\title{
Beyond Eye Care - Low Vision Rehabilitation of a Patient with Recent-Onset Leber's Hereditary Optic Neuropathy: A Case Report
}

Teresa Poon, BSc, OD

T. Labreche, BSc, OD

University of Waterloo

\begin{abstract}
Leber's hereditary optic neuropathy (LHON) is a maternally inherited mitochondrial deoxyribonucleic acid (DNA) mutation that results in painless, sudden-onset, bilateral central vision loss and dyschromatopsia. Currently, there are no proven treatments to prevent or reverse the optic neuropathy in LHON. Accordingly, individualized rehabilitation services and assistive devices for low vision are crucial for helping people with LHON to regain independence and quality of life. This report describes the impact of multidisciplinary low vision rehabilitation on a young man with recent-onset LHON and emphasizes the importance of the provision of emotional support through counselling for low vision.
\end{abstract}

KEY WORDS:

Leber's hereditary optic neuropathy, low vision rehabilitation, multidisciplinary, competitive enablement, counselling

\section{Résumé}

La neuropathie optique héréditaire de Lever (LHON) est une mutation de l'ADN mitochondriale transmise par la mère qui cause une perte indolore et soudaine de la vision centrale bilatérale ainsi que la dyschromatopsie. Il n'existe actuellement aucun traitement éprouvé pour prévenir ou renverser ce type de neuropathie optique. Par conséquent, les services de réadaptation individualisée de la basse vision et les appareils fonctionnels sont essentiels pour aider les personnes atteintes de la LHON à retrouver leur autonomie et leur qualité de vie. Le présent rapport décrit les répercussions de la réadaptation mutilidisciplinaire de la basse vision chez un jeune homme atteint d'un début récent de LHON et met l'accent sur l'importance de la prestation de soutien émotionnel par l'entremise de counselling relatif à la basse vision.

MOTS CLÉS:

Neuropathie optique héréditaire de Lever, réadaptation de la basse vision, multidisciplinaire, habilitation compétitive, counselling 


\section{INTRODUCTION}

Leber's hereditary optic neuropathy (LHON) is possibly the most frequently occurring mitochondrial disease, but its prevalence is still fairly rare, ranging from 1 in 30,000 to 1 in 50,000. Many individuals with the mitochondrial deoxyribonucleic acid (DNA) mutation remain asymptomatic with subclinical changes such as retinal nerve fibre layer thickening and dyschromatopsia. The penetrance in males is $45-50 \%$ and in females only $10 \%$. As a result, approximately $85 \%$ of all individuals with LHON are male. ${ }^{1}$ LHON usually presents in young males between the ages of 15 and 30 years. $^{2}$ Blood analysis showing mitochondrial DNA mutation at one of three possible nucleotide positions is diagnostic for LHON, but this may not be associated with any measurable vision loss. The severity of the vision loss is greater with mutations in positions 3460 and 11778 and milder with mutations in position 14484. These mutations affect subunits of complex I, the first site of the mitochondrial electron transport chain, which leads to decreased adenosine triphosphate (ATP) synthesis and increased oxidative stress and predisposes cells, in particular, retinal ganglion cells, to undergo apoptosis. In LHON, a tendency exists for selective damage to the papillomacular bundle and sparing of larger axons of the periphery. However, the exact mechanism of the selective death of the retinal ganglion cells is still unknown. The loss of retinal ganglion cells leads to pallor of the optic disc with subtle edema and tortuosity of vessels. ${ }^{1,3}$

Several risk factors have been identified in various retrospective studies as triggers of conversion to active LHON in unaffected carriers. These include smoking, exposure to smoke or toxins (ethanol, pesticides, cyanide, and methanol), alcoholism, deficiency of B vitamins, and intake of drugs (ethambutol, aminoglycosides, chloramphenicol, linezolid, zidovudine, and other anti-retroviral drugs) that interfere with mitochondrial respiratory function.,4

A few case reports have shown that an absolute central scotoma in LHON can gradually shrink to a relative scotoma in the year and a half after the onset. In addition, visual acuity can improve as a result of gradual shrinkage of the central scotoma. Good visual recovery occurs even in some cases of LHON with the 11778 mutation, which has the tendency to present with a more severe visual outcome. The mechanism of visual recovery in LHON still remains unknown. ${ }^{5}$

Patients with LHON present with sudden, painless, central loss of vision in one eye, which rapidly progresses to involve the other eye within weeks to months. ${ }^{6}$ Dyschromatopsia is often present as well, with a particular decrease in the perception of red; the mechanism for this is unknown. Patients may describe their vision as being blurry, cloudy, or having a central dark spot. They may also report difficulty with activities of daily living (ADLs). Rarely, Uhthoff syndrome (decreased acuity with increased body temperature) and photopsias (colour sensations) are described. ${ }^{3}$

In the early stages of LHON, fundus examination typically reveals subtle disc edema with surrounding swelling of the retinal nerve fibre layer, circumpapillary telangiectactic microangiopathy, and tortuous vessels. ${ }^{7}$ Over time, mild optic disc pallor and retinal nerve fibre loss result. ${ }^{2}$

Differential diagnoses of LHON include infiltrative optic neuropathy and nutritional or toxic optic neuropathy. Similar to LHON, infiltrative optic neuropathy would also show a thickened optic disc; however, fluorescein angiography will show dye leakage. In addition, magnetic resonance imaging (MRI) will reveal any infiltrative lesions. ${ }^{1}$ Nutritional or toxic optic neuropathy can be confirmed with laboratory serum analysis and urinalysis for various vitamins, folic acid, and pyruvate or investigated through a detailed case history. ${ }^{2}$

\section{CASE REPORT}

D.B., a 23-year-old Caucasian man, presented to his optometrist for a routine assessment to update his spectacle prescription. He reported some blurry vision in his left eye while watching television. The blurry vision had begun rather suddenly and continued to worsen over the course of a few days. He was unaware of any family members with notable ocular or systemic conditions. Other than being a smoker, his medical history was negative for any systemic conditions and medications. 
Figure 1. Red-free photo of the left eye, showing retinal nerve fibre layer loss after 4 months of onset of Leber's hereditary optic neuropathy.

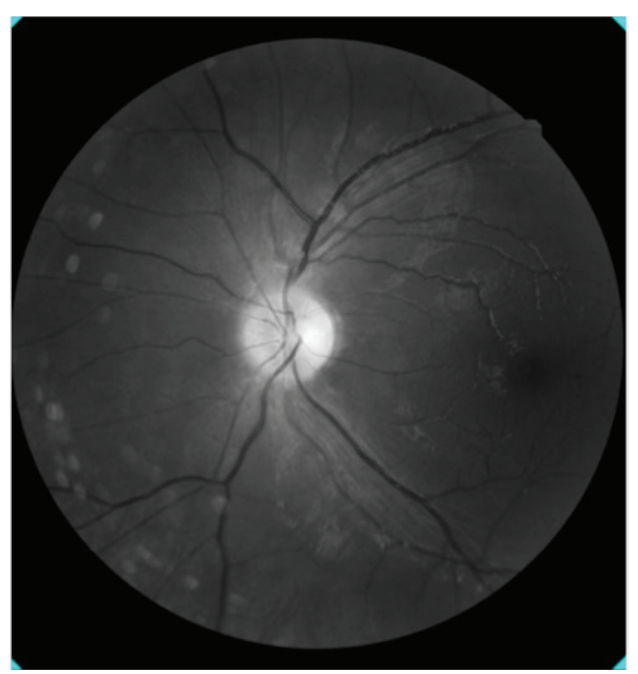

Best corrected visual acuities were reduced to OD (oculus dexter, or right eye) 6/12 (20/40) and OS (oculus sinister, or left eye) 6/12 (20/40). Eye movements were unrestricted, and his pupil responses were normal and without afferent defect. Intraocular pressures were within normal limits by applanation. Cover test showed no strabismus. Colour vision was unaffected when assessed using Ishihara plates. Biomicroscopy results showed normal and healthy anterior segment structures. Dilated fundus examination showed normal maculae with no peripheral pathology OU (oculus uterque, or both eyes). There was edema of the left optic disc, with vascular tortuosity. Due to bilateral unexplained vision loss, D.B. was referred to an ophthalmologist for further workup and laboratory testing. Blood work results confirmed the diagnosis of LHON.

At his 3-month follow-up, best corrected visual acuities were OD 6/12 (20/40) and OS less than 6/120 (20/400). Both eyes appeared straight, and eye movements were unrestricted. Pupil responses were unremarkable and applanation intraocular pressures were within normal limits. Cover test was difficult due to poor fixation, but there was no history of strabismus. Colour vision was reduced to OS 7/9, as determined using Ishihara plates, but remained normal in the right eye. Anterior segment structures were healthy. Fundus photos showed mild optic disc pallor (OS>OD) and red-free photos revealed retinal nerve fibre layer loss (Figure 1).

A month later, his vision in both eyes decreased dramatically to finger counting. Currently, there are no proven treatments to prevent or reverse the vision loss associated with LHON. D.B. was referred to the Centre for Sight Enhancement (CSE) for assessment for low vision.

\section{Management: Low Vision Rehabilitation}

D.B., a carpenter by trade, is an athletic young man who played hockey and soccer. Within 6 months of being diagnosed with LHON, he was unable to participate in any of his favourite sports and he had lost his job. He could no longer see the keypad on debit machines, and he reported that grooming and crossing the street had become progressively more difficult. In addition to these concerns, he was finding each day increasingly long and was finding it difficult to occupy his time. His primary goal was to be able to return to work as soon as possible.

Presenting unaided distance visual acuity was 3/69 OD, 3/91 OS, and 3/69 OU, determined by using a Feinbloom acuity chart. Unaided near-visual acuity was $0.08 / 2.0 \mathrm{M}$ OD, 0.08/2.5M OS and 0.08/2.0M OU, determined by using a Lighthouse Single Letter near-acuity chart. Subjective refraction improved acuity to $3 / 61$ and $3 / 55$ for the right eye and the left eye, respectively. Contrast sensitivity measured with a Peli-Robson chart was reduced to 0.95 OU. Amsler grid testing revealed an absolute central scotoma OU. 
Figure 2. Right Goldmann visual field results illustrating central field loss.

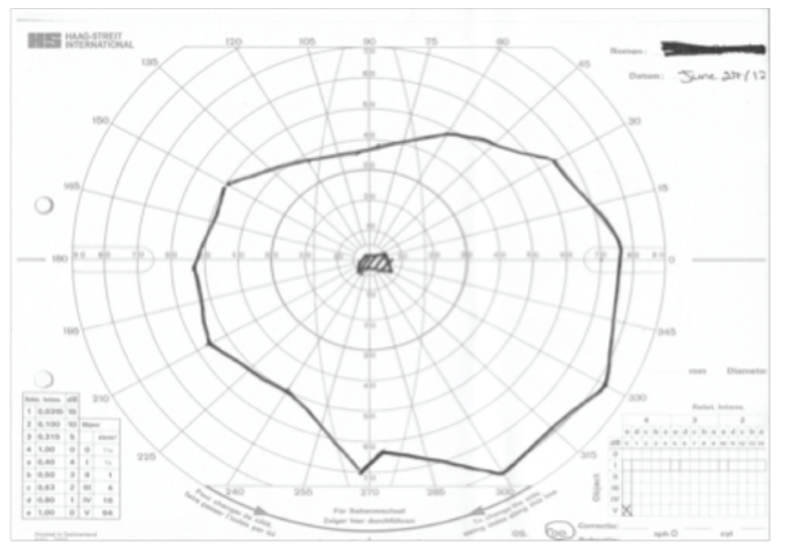

Figure 3. Left Goldmann visual field results illustrating central field loss.

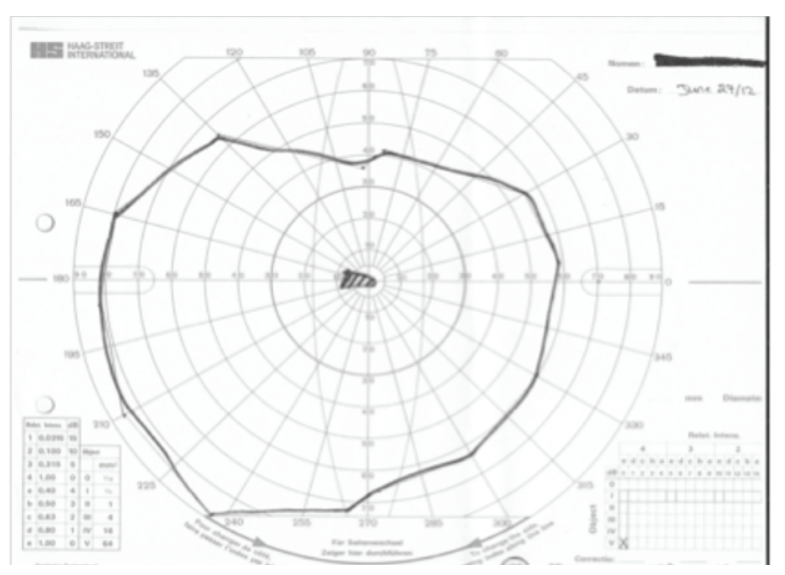

A Goldmann monocular visual field assessment using a V4e target confirmed an absolute central scotoma at about 5 degrees on each side. However, he still had about 130 degrees horizontally and 100 degrees vertically in each eye (Figures 2 and 3).

It was estimated that D.B. would need an equivalent viewing power of $48 \mathrm{D}$ in order to read 1.0M size print fluently. ${ }^{8}$ As a starting point, $24 \mathrm{D}$ near-devices were selected to attempt to achieve survival reading (minimal reading task, as is necessary when reading one's mail) or 1.0M size print at threshold. Following the model of competitive enablement, ${ }^{9}$ several device types were reviewed. A 24D full-field microscope was demonstrated initially, with the microscope over OS and then over OD, which allowed him to achieve a threshold acuity of $1.2 \mathrm{M}$ when the microscope was placed over the right eye. Although unassisted acuity was marginally better in the left eye, this preference for the right eye may have been due to a difference in contrast sensitivity measures between the two eyes. Unfortunately, monocular contrast sensitivity testing was not performed. D.B. was counselled that this device could also be used to aid with eccentric viewing training. He found the spectacles quite heavy. A 24D illuminated hand magnifier was preferred over the full-field microscope. Performance with these devices was compared with his performance while using a portable closed-circuit television system (CCTV). With a CCTV, he readily achieved an acuity performance of $0.5 \mathrm{M}$, and he greatly appreciated the enhanced visibility using enhanced contrast and reversed polarity functions. Ergonomically, he found the increased working distance with a CCTV to be much more effective for sustained viewing activities. 
To address difficulties with TV viewing, the $2.1 \times$ Max TV and $4 \times 20$ Beecher-Mirage binocular telescopes were introduced and subsequently loaned to him to allow him to experiment and decide which one was most suitable for viewing his 46-inch television. Monocular telescopes $(4 \times 12$ and $6 \times 16)$ were demonstrated for distance spotting tasks and as adjuncts to aid mobility tasks such as crossing the street. Orientation and mobility training was recommended.

Although glare was a concern, D.B. did not appreciate any subjective benefits using any of the standard lens tints for those with low vision. Additional appointments were scheduled to follow up this initial assessment and for ADL consultation, a CCTV and high technology assessment, and rehabilitation counselling.

During ADL consultation, the low vision rehabilitation specialist reviewed the assistive devices that D.B. had found most useful from the initial assessment and determined the outcome of the telescopes loaned. Different types of lighting and utilization of colour and contrast were reviewed. The importance of labelling appliances, food, medications, and personal items was discussed along with demonstration of various labelling techniques and devices, including audio labellers. For communication purposes, use of bold lined paper, writing guides, and a black felttip marker with a white board were introduced to increase contrast. Various nonoptical devices that facilitate home safety and overall function such as liquid level indicators and oven mittens that cover the entire arm, magnifying mirrors, devices that can help identify money, and largeface and auditory-output watches and clocks were demonstrated. To provide some leisure activity, $2 \times$ super jumbo playing cards were offered.

The high-technology specialist reviewed several portable and desktop CCTV models and also demonstrated various features on the patient's iPhone $4 \mathrm{~S}$. The iPad was also reviewed for comparison with D.B.'s current Kindle Touch. D.B. appreciated the higher contrast of the iPad, but no significant difference in reading was noted. The speech output function of the Kindle device was also demonstrated and was appreciated by D.B. A separate high-technology assessment to review an assortment of computer adaptations and software (including voice output and OCR software) for both PC and Mac were much appreciated.

D.B. also agreed to an appointment with the centre's low vision counsellor, who provided support services, including career planning and vocation adjustment, information about community resources, and help with applications for financial assistance, in addition to individual and family counselling.

At the completion of the second set of assessments, D.B. indicated that he felt "hope" for the first time. He purchased the jumbo playing cards, a 24-inch CCTV, assistive computer software, and a $4 \times$ Beecher spec-mounted telescope. He had started using the appropriate accessibility features on his iPhone and Kindle reader. He was educated on the Ontario Disability Support Program (ODSP), which offers income support and employment support, and the Ontario Assistive Devices Program (ADP), which provides some financial support toward the cost of devices. ${ }^{10-12}$ Forms were completed to initiate registration for these services. The Ontario Disability Tax Credit form was completed. D.B. was also registered with the Canadian Institute for the Blind (CNIB). ${ }^{13}$

D.B. was provided information about a neurologist who had been doing research on a mitochondrial cocktail consisting of creatine monohydrate, coenzyme Q10, and alpha-lipoic acid, which reduced lactate and markers of oxidative stress in patients with mitochondrial cytopathies. At the time, there was only one randomized, double-blinded clinical trial consisting of 16 patients, so further studies using a larger sample size were still required to clarify potential of this therapy. ${ }^{14}$ After discussing this option, the patient decided that he would like to be referred to this specialist.

Subsequent 6- and 9-month follow-up assessments showed some improvement in contrast sensitivity. D.B. reported continued use of the recommended assistive devices, as well as the strategies and techniques to maximize vision and facilitate ADLs. He had acquired temporary 
employment, but with the help of the counsellor, he was experiencing success in realigning his career goals with his current visual capabilities and was investigating the possibility of further education to achieve these goals.

Our last update from D.B. was via e-mail approximately 18 months after our initial assessment. He was pleased to inform us that he had just recently become employed as a financial advisor.

\section{DISCUSSION}

The Centre for Sight Enhancement (CSE) multidisciplinary team members include optometrists; residents and fourth-year optometry students; a counsellor; a low vision rehabilitation specialist; and high-technology specialists. The CSE model for assessing the appropriateness of device options can be described as a process of competitive enablement. ${ }^{9}$ The goal of the assessment is to determine the best device to enable the patient to perform the identified task instead of simply finding the best device for the task. The patient has the opportunity to explore several device options and choose the device that best facilitates performance of a particular task while considering other factors such as "cost, ease of use, versatility, safety, universality, cosmetics, availability, serviceability, innovation, practicality, and adaptability."

Typically, the desired outcome of an optometric assessment is to address the patient's main objective. Nevertheless, it is important to recognize that low vision rehabilitation is a process similar to any other form of rehabilitation and often takes significant time and effort before an improvement in the patient's quality of life is recognized..$^{15}$ D.B.'s primary goal was to review devices or adaptations that would allow for a speedy return to some form of employment. These are very practical, yet difficult, goals to achieve, considering that, typically, many adults with visual impairments are dependent on others and remain unemployed. ${ }^{16}$ Achieving these goals would require a series of assessments and successful accommodations to improve functional seeing capabilities. D.B. was educated about how vital it is initially to learn the effective use of the remaining vision and the assistive devices prior to re-entering the workforce. This direction often leads to greater confidence with abilities and translates into future employment success and, thus, success in low vision rehabilitation. However, this effort requires the collaboration of all members of a multidisciplinary team. If it is not possible to practise directly with a counsellor, a low vision rehabilitation specialist, and a high-technology specialist, it is prudent to consider a referral, when feasible, to help ensure a successful outcome. ${ }^{17}$

The significance of providing emotional support to individuals with visual impairment has increased, as adjustment to the impairment has been associated with depression and specific personality types. ${ }^{18}$ Family members of the individual with visual impairment can be another strong source of support; however, it is imperative to recognize that they might also experience adjustment concerns and may benefit from counselling. ${ }^{19}$

It is important to inform patients about the services available to them and to introduce them to available support groups for the given condition. This not only benefits the patient by providing further emotional support from those who have experienced a similar visual loss but also allows the patient an opportunity to share his or her story and empower others. The LHON Community provides a detailed list of activities and careers that can be pursued by those with LHON. $^{20}$

Furthermore, the intent of low vision rehabilitation is not to extinguish any hope of future treatment options. As was the case with this young patient, information about research for potential treatment can be passed along to the patient for further investigation.

\section{CONCLUSION}

Although there is no treatment for LHON at the present time, patients with LHON can continue their occupational and social activities. The key is to assess the patient's level of functional vision and provide the appropriate assistive devices. Low vision care should be individualized, taking into consideration the personality of the patient and his or her 
environment. Most importantly, emotional support and counselling are an essential part of the successful care of a patient with low vision.

\section{REFERENCES}

1. Sadun AA, Morgia CL, Carelli V. Leber's hereditary optic neuropathy. Neurol Ophthalmol Otol 2011;13:109-17.

2. Chronister CL, Gurwood AS, Burns CM. Leber's hereditary optic neuropathy: a case report. Optometry 2005;76:302-8.

3. Newman NJ. From genotype to phenotype in Leber hereditary optic neuropathy: still more questions than answers. J Neuro-Ophthalmol 2002;22(4): 257-61.

4. Man PYW, Turnbull DM, Chinnery PF. Leber's hereditary optic neuropathy. J Med Genet 2002;39:162-9.

5. Naka M, Nitta T, Sato I. Long-term evaluation of visual field alteration in Leber's hereditary optic neuropathy with visual recovery. J Neuro-Ophthalmol 2005;29:65-7.

6. Ehlers J, Shah C, eds. The Wills eye manual: office and emergency room diagnosis and treatment of eye disease. Philadelphia, PA: Lippincott Williams and Wilkins, 2008:260.

7. Swartz N, Savino PJ. Is all nondefinable optic atrophy Leber's hereditary optic neuropathy? Survey Ophthalmol 1994;39(2):146-50.

8. Cheong AMY, Lovie-Kitchen JE, Bowers AR Determining magnification for reading with low vision. Clin Experiment Optometry 2002 85(4):229-37.

9. Strong JG, Jutai JW, Plotkin AD. Competitive enablement: a client-centred conceptual model for device selections in low vision rehabilitation. Everyday technology for independence and care. Assist Tech Res Series 2011;29:1033-42.

10. Ontario Disability Support Program, Ontario Ministry of Community and Social Services; 2008. Income support. http://www.mcss.gov.on.ca/en/mcss/ programs/social/odsp/income_support/eligibility/ financial_Eligibility.asp. Accessed June 11, 2012.

11. Ontario Disability Support Program, Ontario
Ministry of Community and Social Services. (2008). Employment support. http://www.mcss.gov.on.ca/en/ mcss/programs/social/odsp/employment_support/ works.aspx. Accessed June 11, 2012.

12. Assistive Devices Program, Ontario Ministry of Health and Long-Term Care. (2008). Visual aids. http://www. health.gov.on.ca/en/public/programs/adp/categories. aspx. Accessed June 11, 2012

13. CNIB, Rehabilitation and Support Services. (2012). Our range of services. http://www.cnib.ca/en/ services/vision-support/range/\#contentstart. Accessed June 19, 2012.

14. Tarnopolsky MA. The mitochondrial cocktail: rationale for combined nutraceutical therapy in mitochondrial cytopathies. Adv Drug Delivery Rev 2008 60:1561-7.

15. Wolffsohn JS, Cochrane AL. Design of the low vision quality-of-life questionnaire (LVQOL) and measuring the outcome of low-vision rehabilitation. Am J Ophthalmol 2000;130(6):793-802.

16. O'Day B. Employment barriers for people with visual impairments. J Visual Impairment Blindness 1999;93(10):627.

17. Strong JG, Pace RJ, Plotkin AD. Low vision services: a model for sequential intervention and rehabilitation. Can J Public Health 1988;79:S50-4.

18. Tabrett DR, Latham K. Adjustment to vision loss in a mixed sample of adults with established visual impairment. Invest Ophthalmol Visual Sci 2012;53(11):7227-34.

19. Bambara JK, Wadley V, Owsley C, et al. Family functioning and low vision: a systematic review. J Vision Impairment Blindness 2009;103(3):137-49.

20. LHON Community. http://www.lhon.org/lhon/ LHON_community.html. Accessed July 16, 2012 